\title{
Frontières
}

\section{Chants pour la maisonnée au chevet du défunt La communauté et l'exil dans les funérailles des Yézidis d'Arménie}

\section{Estelle Amy de la Bretèque}

Volume 20, numéro 2, printemps 2008

Les musiques et la mort

URI : https://id.erudit.org/iderudit/018336ar

DOI : https://doi.org/10.7202/018336ar

Aller au sommaire du numéro

Éditeur(s)

Université du Québec à Montréal

ISSN

1180-3479 (imprimé)

1916-0976 (numérique)

Découvrir la revue

Citer cet article

Amy de la Bretèque, E. (2008). Chants pour la maisonnée au chevet du défunt La communauté et l'exil dans les funérailles des Yézidis d'Arménie. Frontières, 20(2), 60-66. https://doi.org/10.7202/018336ar
Résumé de l'article

Dans les villages yézidis d'Arménie, les funérailles sont un moment particulièrement important dans la vie de la communauté. En même temps qu'elles aident le passage de l'ici à l'ailleurs, elles permettent, de multiples manières, de réaffirmer l'unité et la continuité du foyer (mal) et de la communauté. Omniprésent dans les lamentations, l'exil, littéral ou métaphorique, est toujours lié à un état émotionnel particulier : la douleur de la perte. Cette douleur, qualifiée de " communautaire ", est exprimée notamment à travers les mots, gestes et attitudes des lamentations. 


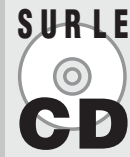

1. Lamentation féminine enregistrée au village de Rya Teze, région d'Aparan, Arménie.

Durée : $7 \min 28 \mathrm{~s}$

2. Çeko. Lamentation enregistrée en studio, composée et chantée par Jono Temuryan. Accompagnement au duduk et synthétiseur.

Durée : $5 \mathrm{~min} 41 \mathrm{~s}$

Résumé

Dans les villages yézidis d'Arménie, les funérailles sont un moment particulièrement important dans la vie de la communauté. En même temps qu'elles aident le passage de l'ici à l'ailleurs, elles permettent, de multiples manières, de réaffirmer l'unité et la continuité du foyer ( $m a l)$ et de la communauté. Omniprésent dans les lamentations, l'exil, littéral ou métaphorique, est toujours lié à un état émotionnel particulier: la douleur de la perte. Cette douleur, qualifiée de «communautaire », est exprimée notamment à travers les mots, gestes et attitudes des lamentations.

Mots clés: lamentation - musique mort - exil - mémoire - lignage - foyer.

\begin{abstract}
In Armenia's Yezidi villages, funerals are particularly important in the life of the community. First of all, they help the passage from the "here" to the "elsewhere". Further, they allow, in multiple ways, the continuity and reaffirmation of the household ( $\mathrm{mal}$ ) as well as of the community in all. The notion of exile, literal or metaphoric, is omnipresent in the laments, and always entails a specific emotional state: pain of loss. This pain is seen as "communal, and is mainly communicated through the laments' words, gestures and attitudes.
\end{abstract}

Keywords: lament - music - death exile - memory - lineage - household.

\section{Chants pour la maisonnée au chevet du défunt La communauté et l'exil dans les funérailles des Yézidis d'Arménie'}

\section{Estelle Amy de la Bretèque, doctorante au Laboratoire d'ethnomusicologie, CNRS UMR 7173,ATER, Université Paris X Nanterre.}

Les Yézidis ${ }^{2}$ du Caucase vivent en majorité en Arménie autour du mont Aragatz. Ils se sont installés en deux vagues dans les années 1828-1829 et 1915-1916 fuyant l'Anatolie orientale ${ }^{3}$.

Le Yézidisme est un système religieux qui a des points communs avec les grandes religions monothéistes ainsi qu'avec les hétérodoxies du Moyen-Orient. Les Yézidis acceptent la réincarnation. Ils croient en un Dieu unique ainsi qu'en sept anges principaux qui peuvent se réincarner périodiquement en personnages emblématiques. Cheikh Adi, pensé comme le fondateur du yézidisme (XII ${ }^{\mathrm{e}}$ siècle) (voir Kreyenbroek, 1995), est vu en prophète.

Commel'écrit Kreyenbroek (Kreyenbroek et Rashow, 2005, p. 6): "Orthopraxy plays a far greater role in the religious life of observant Yézidis than orthodoxy. Knowing one's place (and the resulting rights and obligations), in the complex web of Yezidi social relations can be seen as one of the foremost religious duties. "

Tous les Yézidis appartiennent à l'une des castes endogames héréditaires de Sheikhs, Pirs (chefs spirituels) ou Mirid (disciples) ${ }^{4}$. Chacun des membres de la communauté (y compris les Sheikhs et Pirs) a un Sheikh et un Pir. L'attribution des Mirids est déterminé par un système rigide de relations entre clans et lignages de Sheikhs et de Pirs. Aucune conversion n'est donc possible: on naît Yézidi.

Les Yézidis du Caucase se définissent généralement comme des Kurdes; ils sont kurdophones et présentent de nombreux traits culturels communs avec les Kurdes d'Anatolie. Leur religion diffère cependant, et c'est ce qui les rend parfois plus proches des Arméniens (chrétiens apostoliques). Pendant la période soviétique, l'identité religieuse avait tendance à être effacée et la kurdicité des Yézidis était mise en avant. Depuis la fin de l'URSS, et le conflit armé du Haut Karabakh, la tendance s'est inversée: les Kurdes, par leur identité "musulmane», sont pensés comme des alliés des Turcs et des Azéris. De nombreux Kurdes musulmans d'Arménie ont quitté le territoire à cette période pour l'Azerbaïdjan (les intermariages étaient courants entre Azéris et Kurdes musulmans). Dans l'Arménie indépendante, l'identité yézidie est donc plus souvent mise en avant, à tel point que certains parlent d'un peuple yézidi et d'une langue ezdîkîn.

Les funérailles sont un moment particulièrement important dans la vie du groupe. 

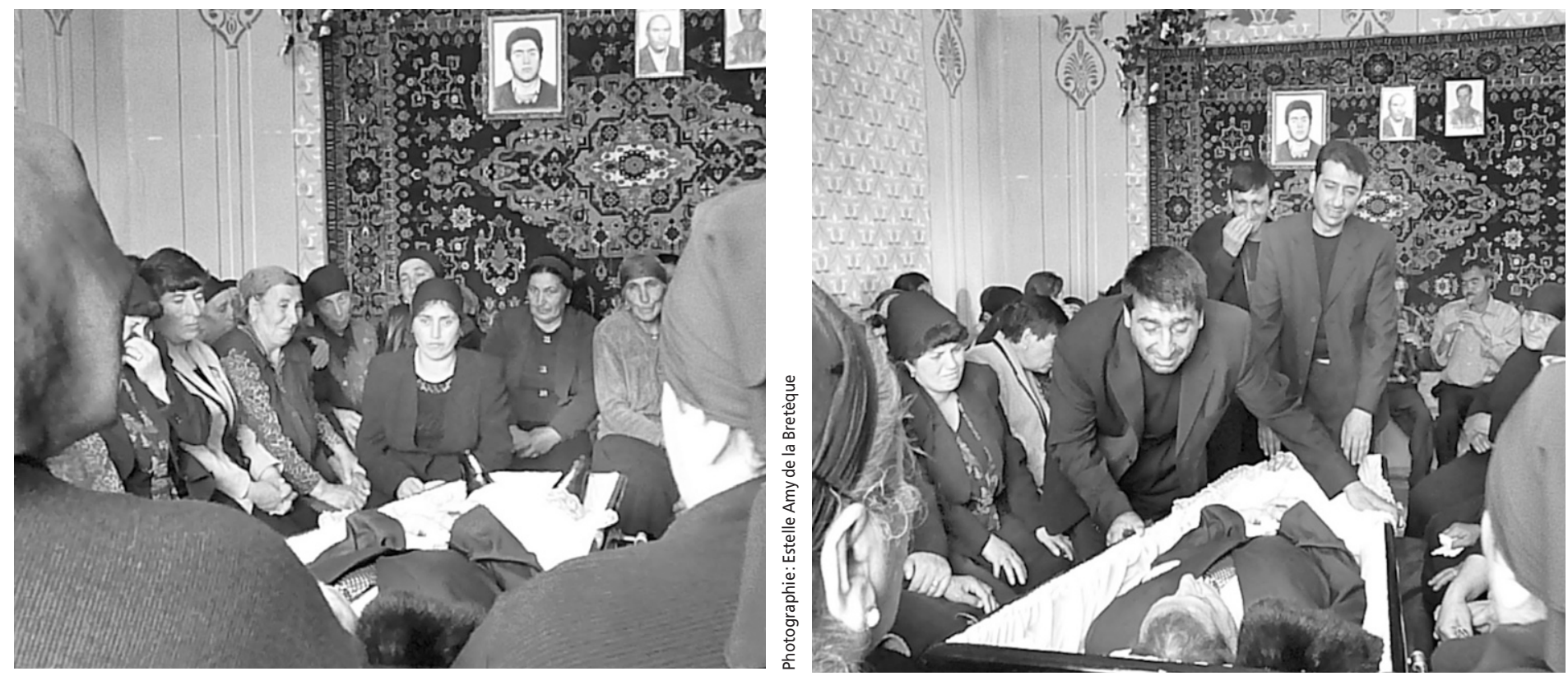

Funérailles de Razmik, village Alagyaz, septembre 2006. À gauche, troisième matinée de veille. À droite, salut des fils à leur père. Au fond de la pièce deux joueurs de duduk accompagnent la scène.

En même temps qu'elles aident le passage de l'ici à l'ailleurs, elles permettent, comme nous allons le voir, de multiples manières, de réaffirmer l'unité et la continuité du foyer $(\mathrm{mal})$ et de la communauté. Le rôle de la parole funèbre chantée, la lamentation, dans ce processus de création, de recréation, d'évolution et d'affirmation du groupe est primordial. Par un ensemble de performances mélodico-textuelles «semiimprovisées» combinées à une gestuelle et une mise en scène codifiées, la mort chantée est l'un des éléments constitutifs de cette refondation.

\section{UNE « GÉOGRAPHIE » SONORE ET ÉMOTIONNELLE DES FUNÉRAILLES: ALAGYAZ, VILLAGE YÉZIDI DU MONT ARAGATZ, ARMÉNIE}

Au matin du troisième jour de veille, le corps du défunt est installé, comme les deux jours précédents, dans une pièce vide de tout meuble. Seuls, sur un mur, des cadres avec les photos des défunts de la famille. Autour du cercueil ouvert, des chaises en arc de cercle entourent le défunt, laissant un espace libre au niveau des pieds. Derrière les chaises, des rangées de bancs sont installées en arcs de cercles concentriques (autour du cercueil). C'est à partir de 9 heures, au matin du troisième jour, que les femmes ${ }^{6}$ du village s'installent sur les chaises et les bancs. Les femmes qui chanteront s'assoient au plus près du cercueil autour de la tête du défunt, ce sont elles qui guident cette dernière matinée de pleurs chantés, ce sont elles qui sont responsables du crescendo émotionnel menant à la levée du corps. Ces femmes sont des parentes proches.
Par leurs lamentations, elles arracheront des larmes aux participantes assises derrière elles sur les bancs. Ces dernières ne chantent pas, ou rarement, mais elles participent à l'univers sonore par leurs pleurs, gémissements, cris et sanglots. Au pied du cercueil, l'espace libre accueille par intermittence les hommes qui viennent saluer le défunt, silencieusement, en s'exclamant birao birao (frère, frère) ou parfois (en particulier lorsque le défunt est un homme) en chantant. C'est dans cet espace aussi que les hommes religieux de la communauté (Sheikh et Pir) récitent les Qewl et Beyt ${ }^{7}$ indispensables à ces cérémonies funèbres ${ }^{8}$. Les hommes ne restent dans la pièce que le temps de leur énonciation.

Le centre sonore est donc concentré autour du cercueil, de même que le centre de la parentèle féminine du défunt (d'abord les consanguins, puis les collatéraux). La gestion de l'espace entourant le défunt nous donne une première image sonore, mais aussi visuelle, du foyer ( $\mathrm{mal}$ ), nous permettant de distinguer «nous» et «les autres». Les regards des participantes sont dirigés vers les parentes du défunt situées au plus proche du cercueil: ce sont la mère, les sœurs, les filles et les belles-filles désignées par le terme dilsewat: « au cœur brûlant». Cet épicentre émotionnel et sonore s'oppose à une périphérie plus passive et moins brûlante. Cependant, si les lamentations viennent toujours du centre, elles secouent aussi la périphérie.

Dans cette dernière matinée précédant l'enterrement, les lamentations se suivent sans interruption. Les voix sont éraillées par deux jours de pleurs. La tension monte, la pièce est de plus en plus bruyante, certaines femmes se frappent, d'autres se griffent le visage, quelques évanouissements provoquent cris et agitation. Les derniers parents, venus parfois de Géorgie ou de Russie sont arrivés; toutes les femmes du village se serrent sur les bancs.

Les lamentatrices s'adressent au défunt, elles l'accusent de faire souffrir les vivants par sa mort. Elles rappellent les faits marquants de la vie du défunt, racontent sa dernière journée, qualifient sa mort d'injuste, le somment de se réveiller:

\section{Ax, de birîna kekê Samo k'ûre, pir xedare, k'ew lê naê,}

Ax, de emê dikin-nakin ç'are nabe,

$A x$, de rave, rave, H'esenê bira, de tu rave,

Ax, de gelo bêy te h'ale malxwêtya mala giran wê çawabe ${ }^{9}$.

Ô, la blessure du père de $\mathrm{Samo}^{10}$ est profonde, dangereuse et ne cicatrise pas,

Quoi que nous fassions, cela ne sert à rien,

Lève-toi, lève-toi, frère Hesen, lève-toi,

Sans toi, qui va prendre la place de chef de famille, d'une grande famille.

Les pleureuses s'adressent aussi aux participantes, elles chantent les morts de chacune des femmes de l'assemblée. Elles les nomment à tour de rôle et rappellent leurs souffrances: "Ô Filet, comme ta maison est triste depuis que ton fils est mort. » Parfois elles parlent en leur nom, comme si ces femmes étaient en train de chanter ou parler. La pleureuse peut aussi 
rappeler les différents morts récents du village dans une même phrase rappelant la douleur commune à toutes :

Gelî omid-êşano, hetanî xweşbim rûbarê vê dinyâ

Dilê minê usa bikale

Dîdeme xweş dîdeme, gelî omid-êşana, ez we gişkara divêjim

Bextê weme, bextê kula dilê we h'emyame

Herç'î nexweşî̂ kulêne, herç'î qurbanê avêne, herç'î kuştîê k'êrêne

Dilê wan șewitîye, dû-dermanê dox'tira t'û ç'arê wan nake 11

Ô, proches, chers, tant que je suis vivante sur cette terre

Mon cœur bêlera

Une si belle allure, mes chers et proches, je vous le dis à tous

Je vous le jure, la douleur nous est commune

Certains souffrent d'un cancer, d'autres sont tués par la vodka, d'autres encore par un couteau

Ils vont mal, les médicaments n'aident pas.

Parfois au lieu de mentionner un nom, l'orateur désigne quelqu'un par son lien de parenté (frère, père, mère), et cette évocation fait écho chez presque toutes les participantes. "Qu'il est dur de perdre son fils, centre du foyer.»Voici un exemple chanté par un Sheikh lors des funérailles de Razmik, chef de famille vivant à Moscou:

Wey, mirina te raste, ne derewe

Ser tera sekinye komeke giran, wê ax bike bi dil şewate

Wey, birao, birao

Hay birao, hay birao

Heylo birano, biratî şîrine

Me'nekî k'otelê syara nagerîne, gemê dikoje, hêsira dibarîne

Ay de gelî bira, Dewrêş bira, mirin e'mrê Xwedêye

Lê ku tina bira dilê birada birîne dimîne, ay birao, ay birao ${ }^{12}$.

Ô ta mort est réelle, ce n'est pas un mensonge

Elle a cassé le dos de ta famille et l'union de ce clan

Ô, frère, frère

Ô, heylo, heylo, frère,

Heylo, mon frère, la fratrie est si douce,

Ne sortez pas le cheval, avec les beaux habits du défunt, il ne mord pas, mais il pleure amèrement ${ }^{13}$.

Ô, frère, frère Dewreş, la mort est un ordre de Dieu

Mais le meurtre d'un frère restera une blessure dans le cœur de son frère, ô frère, frère.

irin

Dans cet extrait, les paroles sont assez générales, nombreuses sont les femmes qui connaissent des situations semblables, la lamentation aura ainsi un impact plus grand, provoquant pleurs, cris..

Parfois encore la lamentatrice prend la place du défunt, le fait parler, établit des dialogues entre les vivants et les morts. C'est le cas de l'extrait ci-dessous qui fait référence aux tresses que les femmes enterraient avec le corps de leur père, frère ou mari.

Gulîbirno, mi go hevra bêjin «bavo, bavo, bavo, ç'evê me jî lê têda mao»

Malxuê digo, "la-lao, ç'evê min jî têda mao ${ }^{14}$.

Ô, celles aux tresses coupées! Dites en chœur «Père, père, père, père, nos yeux sont restés dans son gilet».

Le chef de famille répond: " mes enfants mes yeux aussi sont restés dans le gilet».

Le rôle central du fils, du frère et du père, ressort à la lecture de ces quelques extraits. Dans une tradition patrilocale, le fils en particulier est la garantie de survie d'une lignée. Les funérailles des hommes sont toujours beaucoup plus sonores que celles des femmes: plus de lamentations, plus de pleurs, de cris, plus de monde que dans les funérailles des femmes. Comme si, par la perte d'un fils ou d'un frère, c'était le foyer entier qui était en péril et, par extension, la communauté tout entière.

À la fin de la dernière matinée de veille, on procède à la levée du corps. Vers $13 \mathrm{~h}$, les hommes entrent dans la pièce en

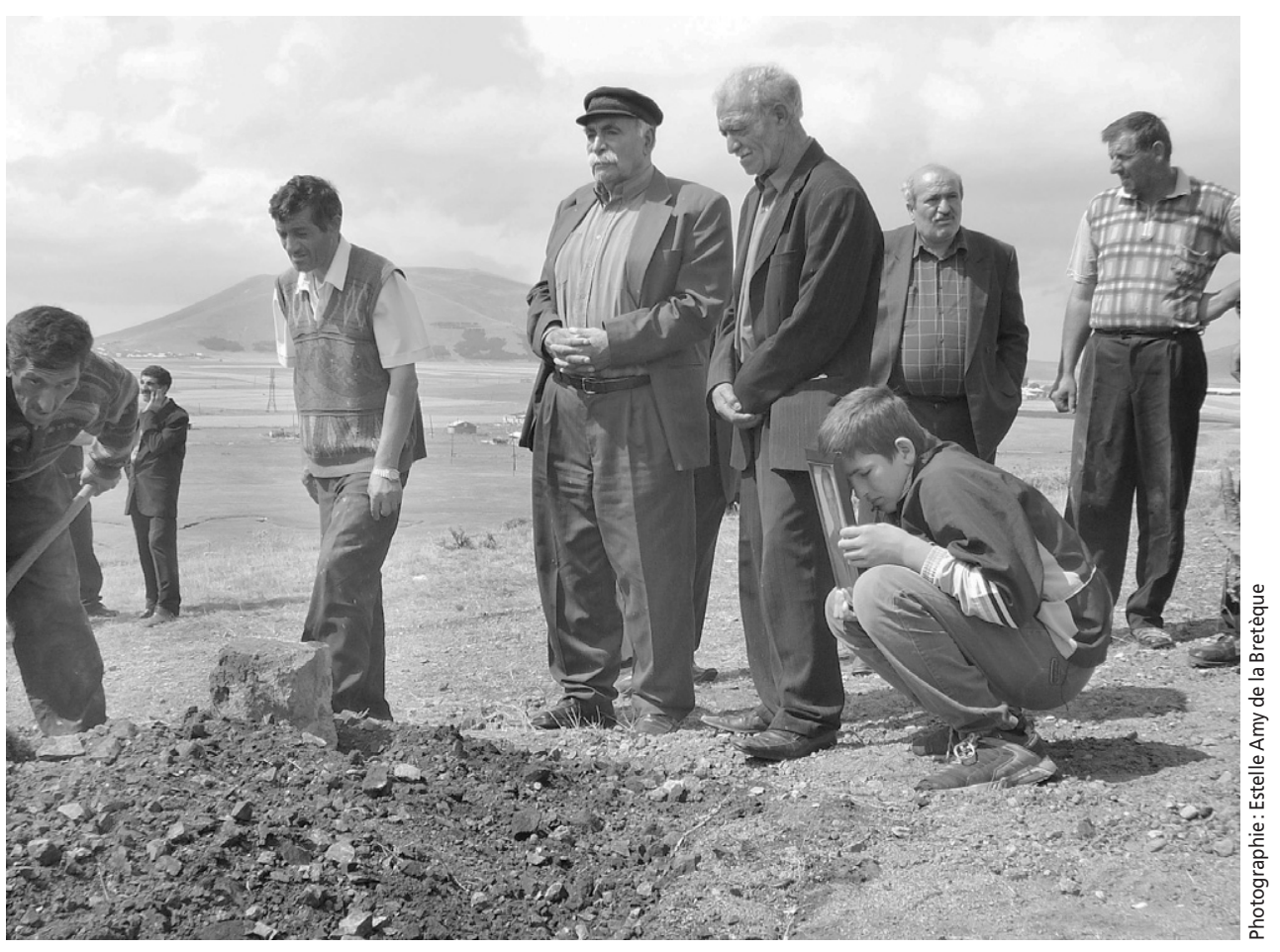

Funérailles de Razmik, village d'Alagyaz, septembre 2006. Sheikh Xalo et Sheikh Gno récitent des prières tandis que les hommes referment la tombe. demandant aux femmes de sortir. Les participantes sortent à l'exception des plus proches parentes «au cœur brûlant» qui se trouvent au plus près du cercueil. Montrant leur refus d'abandonner leur proche, elles se lamentent de concert en se penchant sur le cadavre, en l'embrassant. À ce moment, les voix sont très tendues, presque criées. Les hommes font sortir les femmes de la pièce en les tirant, traînant, portant celles qui ont perdu connaissance. La pièce devient silencieuse, on entendra juste la voix du Sheikh qui chante une prière, et les cris étouffés des femmes, dehors, qui se lamentent. Le cercueil est ensuite sorti de la maison et installé dans la cour où tous les proches parents, hommes et femmes, se lamentent. Puis la procession vers le cimetière commence. Â l'avant, le Sheikh (ou les Sheikhs) chante des prières; ensuite vient le cercueil, porté par de proches parents masculins, puis parfois des joueurs de $d u d u k$ (hautbois) suivis par les hommes silencieux, et enfin, les femmes se lamentant. Au cimetière, les lamentations des femmes continuent jusqu'à la fermeture du cercueil et sa descente dans la fosse. À ce moment, les femmes quittent l'assemblée, s'éparpillent et se dirigent vers leurs tombes familiales, entamant des lamentations pour leurs proches. Le cimetière tout entier résonne alors de lamentations individuelles mais simultanées, pour les défunts de la communauté. Les hommes, pendant ce temps, referment la tombe et écoutent le Sheikh réciter des prières. Ils mangeront ensuite sur la tombe en trin- 
quant à la vodka pour les défunts de la communauté. Les femmes mangent de leur côté, plus silencieuses.

\section{LES TROIS EXILS CHANTÉS}

Dans les lamentations funèbres, trois grands types d'exil sont évoqués et mêlés : l'exil du mort, l'exil de la communauté et l'exil des femmes.

La mort est un départ vers un autre monde: le défunt quitte la communauté, quitte son foyer. La mort est ainsi, sous plusieurs aspects, vécue comme un exil. Il est très courant de mélanger mort et exil dans les lamentations. Lorsqu'un membre de la communauté meurt en exil il est, dans la plupart des cas, enterré au village: son corps est, comme nous l'avons vu, ramené, parfois de très loin. Ce retour est célébré dans les lamentations comme un retour d'exil, qui est cependant immédiatement suivi par un départ vers un nouvel exil.

Go xûşkê bese tu bigrî, bikewgirî,

Hela der-dorê xwe binihêre gelek hene,

Hela çerxa vê mirinê hatye dora me jî,

Wey lê lê lê, hey lê xûşka mine bêbavê,

Bavo, bavo, xerîvo, rê dûro, bavo, bavo,

Bavê me saxe ji xerîbîê hatye,

Wê dîsa sivê berê xwe bide xerîbîe,

Wê here îdî nầ, naê.

Tu as assez pleuré sœur, assez pleuré amèrement,

Regarde autour de toi, beaucoup sont comme toi,

La mort a aussi frappé notre maison/ foyer,

Oh, ma sœur orpheline,

Père, père, tu es au loin, en pays étranger,

Notre père est rentré vivant de l'exil,

Demain il retournera en exil,

Il partira, et ne reviendra plus ${ }^{15}$.

Xerîb ${ }^{16}$, xerîbîe, xerîbîstan: ces différentes variations de l'exil/étranger/terre étrangère sont présentes quotidiennement dans les conversations des Yézidis d'Arménie. L'exil peut désigner des situations et états divers, il peut être littéral ou métaphorique, mais il est toujours lié à un état émotionnel particulier: la douleur de la perte. Selon les situations $x e r i ̂ b$ peut être ce qui est en dehors de la maisonnée, en dehors du village ou du groupe de villages ou parfois hors d'Arménie. Depuis la fin de l'URSS, les Yézidis ont massivement émigré, notamment en Russie et en Allemagne, en raison de la crise économique qui a frappé l'Arménie. Moscou est par exemple caractérisée de «maudite» dans les lamentations: elle est la ville pour laquelle tant de villageois ont quitté leur foyer.

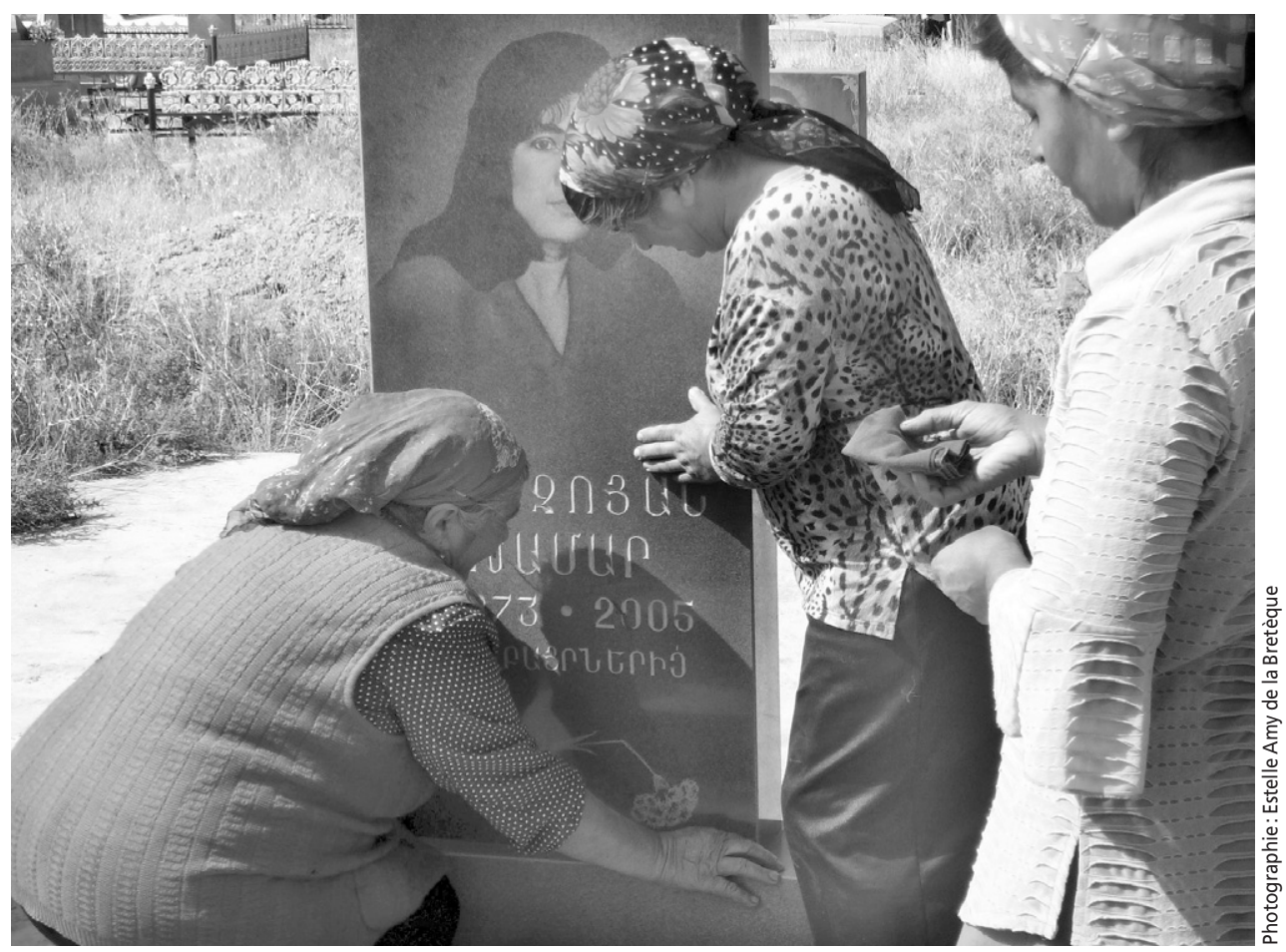

Cejna Mazala, fête des tombeaux, village de Feriq, septembre 2006. Les femmes se lamentent sur les tombes familiales.

Wey xerîbo, hevt sala min nedîto, nebînao,

Min dît delalê dilê min, rya Ûrisêta şewitî de'vî-de'vî,

Qurbana canya teme, Moskva wêran bêdabet $\hat{l}^{17}$

Oh, exilé, depuis 7 ans je ne t'ai pas vu, pas entendu,

Mon cœur, que les routes de Russie soient maudites,

Je me sacrifierai pour toi, la maudite Russie apporte le malheur.

En même temps qu'elles permettent le passage du mort de l'«ici » à l'« ailleurs », les funérailles entraînent le retour des membres "exilés» de la communauté. La lamentation suivante illustre cet appel des exilés à rentrer au village pour les funérailles. Le ton est un peu héroïque: les deux défunts sont morts sur le front du Karabakh au début des années 1990.

Feleka xayîn, te çima wa kir,

Mala me xiravkir, ocaxê me korkir,

Dilê dijmina, neyara me şakir,

Ax, de çawa Valodê bira sal temam nekir, E'lîe bira birîna me tezekir.

Feleka xayîn careke dinê serî h'ejand, ç'eplê xwe me barkir,

De go gelî birano, bikin, bilezînin, caveke nebixêrîê xerîbîêra bişînin,

De bêjin Feyzo, malxiravo, Mîşa, ocaxkoro, nesekinin werin,

Hevt sal Lêyla destê Tîtalê bira bigrin ser mala bavê xweda po man vegerin,
Mala bavê min mala mêra bû, mala șêra bû,

Hewara me navê Xwedê, kê dîtye, kê bînaye,

De çawa nava serê salekêda hev belabu $\hat{u}^{18}$

Traître destin, pourquoi as-tu fait cela,

$\mathrm{Tu}$ as détruit notre maison, éteint notre foyer,

Le cœur de nos ennemis s'est réjoui, malveillant,

Ô, pourquoi Valod n'a-t-il pu attendre la fin de l'année, et frère Ali a ravivé notre plaie,

À nouveau le destin fatal a secoué la tête et menacé de la main,

Dépêchez-vous, frères, envoyez la mauvaise nouvelle en exil,

Dites aux malheureux Feyzo et Mîşa: venez vite,

Que Lêyla, mariée depuis 7 ans, prenne Tîtal par la main et rentre à la maison paternelle.

La famille de mon père était une famille d'hommes, une famille de lions,

Ô, Dieu, qui a vu, qui a entendu,

Comment en un an la famille s'est effondrée.

Être $x e r i ̂ b$, séparé des siens est tragique, au moins pour ceux qui restent au village. La communauté se reconstruit autour des va-et-vient lors des mariages, mais surtout des funérailles. La fête des tombeaux (cejna mazala) est aussi une fête majeure dans le 
calendrier et tous font leur possible pour être dans leur village ce jour-là. Cejna Mazala a lieu une à deux fois l'an, au printemps et parfois à l'automne, au moment du départ et du retour des pâturages estivaux. Ce jour-là, tous se rendent au cimetière. Chaque foyer a son espace dans le cimetière, tout comme au village. Sur les tombes familiales, les femmes se lamentent, les Sheikhs et Pirs disent des prières, puis un repas est partagé. Ainsi, ces lamentations individuelles deviennent collectives du fait que tout le cimetière se lamente en même temps. Il se produit de même, lors des funérailles, au moment où les hommes enterrent le corps du défunt. Dans ces lamentations sur les tombes familiales, les femmes annoncent aux défunts le départ et le retour, concrétisant ainsi l'ici et l'ailleurs, le mal (foyer) et le $x e r i ̂ b$ (exil).

La notion d'espace familial et communautaire est très présente dans les mots et les attitudes de ces lamentations, simultanées bien qu'individuelles. Définissant un espace communautaire d'exilés autour de la mémoire des absents et des morts, l'expression individuelle crée, dans ce cas, un sens de la collectivité par la simultanéité des lamentations.

Les habitants du village désignent souvent ceux qui ont migré comme «nos villageois», ainsi l'unité locale «le village» transcende la localité physique. Quand les hommes adressent, à un homme défunt, les exclamations birao birao (frère, frère), ils indiquent que la personne qui va être enterrée est un frère, l'un des nôtres: il doit être enterré avec les siens. Être enterré parmi les siens, chez soi, est vécu comme un acte ultime de l'appartenance à l'entité villageoise.

Par ailleurs on peut trouver dans certaines chansons la mémoire d'un exil initial d'Anatolie. Dans la plupart des cas, Kars est donné comme ville d' «origine». Je pense que ces paroles sont plus présentes dans la région de Talin, probablement en raison de leur cohabitation avec les Sassountsi ${ }^{19}$ qui sont connus pour leur extrême nostalgie envers leurs villages dans la région de Sassoun (actuelle Turquie). Il est aussi possible que la géographie renforce et guide la mémoire: dans ces villages, proches de la frontière, les montagnes de Turquie sont omniprésentes dans le paysage quotidien. Les villageois disent de ces montagnes : notre terre. Cette terre devient alors un rappel constant d'un espace qui, aujourd'hui, est hors d'accès.

Le dernier exil chanté dans les lamentations est celui traditionnellement vécu par les villageoises yézidies après leur mariage (comme c'est le cas dans de nombreuses communautés du Moyen-Orient). Les femmes quittent à leur mariage le foyer pater- nel pour aller vivre dans la maisonnée de la famille de leur mari. Cette expérience est vécue comme un exil. Les femmes disent alors vivre entourées d'étrangers. C'est souvent une expérience douloureuse: le statut de la femme est celui d'une étrangère auprès des beaux-parents auxquels elle se doit de ne pas adresser la parole au moins jusqu'à ce qu'elle donne naissance à un fils. Elle stabilise alors sa place dans ce nouvel environnement.

Durant les cérémonies de mariage, il n'est pas rare d'entendre la mère de la fiancée chanter une lamentation pour sa fille qui quitte le foyer paternel ( $\mathrm{mal}$ ) afin d'aller vivre en exil (xerîb/xerîbîstan). La fiancée elle-même pleure généralement pendant toute la danse (govend) avec son mari et les membres de sa famille - une ronde symbolisant le passage d'un foyer à un autre. Les lamentations funèbres parlent souvent de cet exil féminin : une mort dans la famille altère la composition du foyer. Par exemple, quand le chef du foyer meurt, ses belles-filles peuvent chanter: «Le père de mon foyer nous a laissées, ô, je suis à nouveau étrangère, je suis en exil. » Lorsqu'une mère sans fils meurt, les lamentations parlent d'une double mort car elle ne laisse derrière elle aucun descendant qui permettrait au lignage de survivre - même si elle a beaucoup de filles, puisque toutes appartiennent au lignage de leur mari ou futur mari.

Ne bave, ne birangê dayka me h'elale Dûrî serê hersê qîza warê wêyî reşe [...]

Ç'evê minê rênga xerîbê teda maye

[...]

H'izret xaê kire gazî, go "Kulîlk lao, go ezê xerîbim, xerîba vê Cizîrê » ${ }^{20}$

Notre mère n'a ni père, ni frère

Que Dieu protège ses trois filles, elle n'a pas de fils

[...]

J'attendrai à jamais ton retour d'exil

[...]

Sœur Xazrat a hurlé : « Kulilk, fils, je suis étrangère, étrangère dans ce Cizire. »

Ces trois types d'exil sont présents, à la fois dans les mots des lamentations et dans les attitudes adoptées par les participants dans les funérailles. Le fait musical total qu'est la lamentation (Rudenko, 1982, p. 10) lie mort et exil.

\section{LA MORT CHANTÉE, ENTRE EXPRESSION IMPROVISÉE ET CONSTRUCTION DE MÉMOIRE COLLECTIVE}

Les lamentations, qui peuvent être de longueur très variable, sont fragmentées au niveau du texte, comme s'il s'agissait d'une série de flashs. Ceux-ci font, cependant, sens pour l'auditoire puisque ces fragments et flashs évoquent des personnes, actions, faits et objets connus de tous.

Les pleureuses affirment dans tous les cas «dire des paroles» (tu kilame dibêje) et non pas chanter. Elles ne se définissent pas comme des chanteuses. Les mots «chant» et «chanter» sont réservés à des formes plus fixes au niveau mélodique et métrique, souvent des pratiques collectives, comme par exemple certains chants de mariage.

Les lamentations sont improvisées sur un schéma mélodico-rythmique récitatif extensible en fonction du nombre de syllabes $^{21}$. Il est intéressant de noter que les mélodies, rythmes et paroles des lamentations ne sont pas spécifiques au répertoire funèbre: on retrouve de nombreuses similitudes dans d'autres répertoires traditionnels, en particulier dans les chants de type épique des bardes dengbêj (littéralement: ceux qui disent la voix). Ainsi les constituants primaires d'une lamentation sont peut être plus liés au moment même où celle-ci est prononcée, aux émotions en présence à ce moment précis, à la gestuelle qui l'accompagne, etc., qu'à des caractéristiques formelles de la mélodie ou du texte.

Si les schémas mélodico-rythmiques traditionnels ne sont pas spécifiques à ce répertoire, ils correspondent cependant à un certain type d'énonciation: les lamentations sont l'expression des sentiments d'une personne à un moment donné. Les lamentatrices affirment créer sur l'instant, même si elles se basent sur des schémas mélodiques et textuels existants. Ce type d'expression chantée d'une douleur accompagne souvent les activités de la vie quotidienne des femmes. Les jeunes mariées bercent souvent leurs nouveau-nés avec de telles lamentations. (Rappelons qu'il est interdit à la bru d'adresser la parole à ses beaux-parents, au moins jusqu'à la naissance d'un fils.) «C'est en disant des lamentations qu'on arrive à s'exprimer. Les mots tout seuls, ça ne marche pas, ça ne m'aide pas. Après avoir dit une lamentation je me sens soulagée» (Zîne, Alagyaz). En certaines occasions, on peut préférer la lamentation à la voix parlée pour s'exprimer, en particulier pour les femmes... le message produit transmet alors des informations plus complexes à l'auditeur ${ }^{22}$.

Dans les funérailles, ce qui semble important est de participer. C'est comme si chacun à tour de rôle contribuait à l'émotion collective et à la performance collective (au sens large du terme), avec sa propre expérience, son opinion, son information ou sa façon de vivre l'émotion de la perte.

Par le rappel des défunts et des absents, les lamentations sont aussi bien sûr un acte 
de mémoire contre l'oubli. Oublier un mort est oublier un lignage, c'est-à-dire perdre un bout de soi. La fête des tombeaux (cejna mazala) est, en ce sens, un jour primordial dans le calendrier des Yézidis de Transcaucasie et tous les membres de la famille font de leur mieux pour être présents, même s'ils doivent venir de très loin. L'importance accordée à cette fête montre la place des morts dans la construction du groupe. D'autres fêtes calendaires sont d'ailleurs liées à la mémoire des morts, comme Xidirnebi ${ }^{23}$ en février et Kiloç ${ }^{24}$ en mars.

Aujourd'hui ces actes de commémoration des morts utilisent parfois un registre différent: ils peuvent être enregistrés, filmés et parfois commercialisés. On trouve en effet sur les marchés d'Erevan des lamentations sur format mp3, chantées par des chanteurs professionnels et accompagnées d'instruments de musique (essentiellement $d u d u k$, synthétiseur et violon). Ces lamentations illustrent notamment des morts exemplaires: celles de soldats Yézidis décédés sur le front du Karabakh par exemple, ou même des lamentations commandées pour des chefs de gangs mafieux, basés à Moscou. Ces enregistrements contribuent alors à la création d'une mémoire collective plus homogène et plus fixe. Cependant ce principe n'est peut-être pas si nouveau: avant l'époque des enregistrements une belle lamentation pouvait être reprise dans d'autres contextes, se transformant petit à petit en chants de héros (kilame ya meraniye). Le mort exemplaire peut produire des chansons qui vont être chantées à d'autres moments de la vie. Cela renouvelle le groupe et l'appartenance au groupe avec de nouveaux héros.

Hey lo bavo,

Neyar, xêrnexazê Ç'ekoê Xidir qeremanê e'şî̂ gele, gelek hene,

De eva sê roje, sê şeve wêrana bajarê Moskvaêda hev civyane,

De dikin şêwra ku tina Ç'ekoê Xidir, ze'vê sê salane.

Go mêrkujê, bêxwedêyo,

Nevî, nevî berê xwe didî ç'eka, gulle û fişekê ser ç'eka bêne ç'are, wey, êtîmo,

De agirê wî tûjin mînanî şûrê wan kewana, ewî xortekî cihale, e'mrê wî bîstşeş sale,

De ewê ya bi Xwedê mînanî fermana Rostemî Zale,

$[\ldots]$

Hewarîê Ç'ekoê xweşmêr weke lê-lê miştê k'ûçe $\hat{u}$ meydanê,

Dewir-zemanada ser t'u merî nebûye $\hat{u}$ neqewimye

De çawa îro hatye kirinê ser Ç'ekoê Xidir, şêrê çargurç'ikî notlanî

Rostemî Zale.
Ô, père,

Çekoê Xidir a beaucoup d'ennemis malveillants.

Cela fait déjà trois jours et trois nuits qu'ils se sont rassemblés dans la maudite Moscou,

Ils discutent du meurtre de Çekoê Xidir, marié depuis 3 ans.

Ô, assassin, ô, homme sans foi/Dieu,

Ne lève pas ton arme et ne l'utilise pas, ô, maudit,

Son feu est si tranchant, tel une épée, il est si jeune, il n'a pas guère que 26 ans.

Mais il a l'ordre de tuer, un ordre comme venant de Rostemî Zale,

[...]

Aux funérailles de Çeko il y avait une mer de monde,

Depuis la nuit des temps il n'y avait eu un deuil pour aucun homme,

Tel celui d'aujourd'hui aux funérailles de Çekoê Xidir, lion sans peur, comme Rosteme Zal.

De plus, l'insertion parfois d'un duo de duduk qui accompagne un chanteur professionnel invité pour les funérailles (et rémunéré) est une nouveauté musicale majeure. Cette pratique, répandue chez les Arméniens de la région, semble avoir gagné les villages yézidis depuis une dizaine d'années. Faut-il y voir le signe d'un alignement sur les traditions arméniennes dans un contexte post-soviétique de renouveau nationaliste? Il est important de noter que, pour le moment au moins, on ne fait appel à ce chanteur professionnel que lors de la mort d'un homme. Les lamentations de ce chanteur sont toujours sur le même modèle mélodico-rythmique, mais sa technique vocale est beaucoup plus élaborée, et ses couplets sont alternés avec des réponses au duduk (un duduk ornemente tandis que l'autre tient le bourdon). Sa performance ne convient pourtant pas à certaines pleureuses dans la salle de veille du corps : « les duduk font pleurer, c'est triste, mais le chanteur, il ne sait pas vraiment quoi dire, il ne sait pas comment faire pleurer, comment toucher au cœur, comment faire sortir la peine », commente Hbo, pleureuse dans toutes les funérailles du village d'Alagyaz depuis maintenant 10 ans que son fils est mort. Le chanteur dont elle parle vient pourtant d'un village voisin et il connaît toutes les femmes de l'assemblée. Mais, d'après $\mathrm{Hbo}$ et les femmes de l'assemblée qui acquiescent en hochant la tête: «il n'a pas la peine en lui ».

Les exils chantés semblent être un élément-clé dans la construction d'une idée de soi et des autres. Les funérailles sont des rituels de commémoration des morts et de redéfinition des relations entre les morts et les vivants. En ce sens, ils permettent le rétablissement du groupe, en grande partie éparpillé, et la redéfinition des notions et des rôles de chacun en fonction de son genre et de sa classe d'âge.

Véhiculant des émotions, telles la souffrance ou la nostalgie, ces chants sur l'exil et la mort définissent des frontières entre nous et les autres et entre ici et ailleurs. Par l'analyse du filtre du discours codifié qu'est la parole chantée, cet article a essayé de montrer les dynamiques territoriales et temporelles entourant la mort et l'exil.

Ce fait musical complexe, qui permet le départ du défunt, véhicule des traits communautaires qui se résument dans les mots de Sûté: "Je n'avais pas vu mes frères depuis sept ans, je n'avais pas vu leurs fils, je n'avais pas vu mes sœurs, mes cousins, neveux... Tous sont rentrés d'exil pour pleurer mon fils. La peine de mon cœur ne s'assouvit pas. Ah, exil fatal, c'est en pleurant nos morts que l'on survit, c'est en chantant nos morts que les Yézidis existent. »

\section{Bibliographie}

ALLISON, C. et P.G. KREYENBROEK (1996). Kurdish Culture and Identity, Londres, Zed Books.

KREYENBROEK, P.G. (1995). Yezidism : Its Background, Observances and Textual Tradition, New York, Lewiston.

KREYENBROEK, P.G. et K.J. RASHOW (2005). God and Sheikh Adi are Perfect. Sacred Poems and Religious Narratives from the Yezidi Tradition, Wiesbaden, Harrassowitz.

OMERXALÎ, X. (2006). «Cejna Xidir Nebî û Xidir Eylas cem êzîdiyên Ermenîstanê » (la fête de Xidir Nebî et Xidir Eylas chez les Yézidis d'Arménie), Ûrkêş, $n^{\circ} 1$, Gävle (Suède), p. 16-28.

RUDENKO, M.B. (1982). Курдская Обрядовая Поэзия: Похоронные Причитания (La poésie rituelle kurde: lamentations funèbres), Moscou, Naua.

\section{Notes}

1. Cet article est basé sur des recherches de terrain menées entre 2005 et 2007 dans les villages yézidis de la région du mont Aragatz en Arménie (région d'Aparan, Talin et Hoktemberian)

2. Les Yézidis sont estimés à 60000 en Arménie et Géorgie, 15000 en Syrie. En Irak, les sources varient de 120000 à 250000 . Les Yézidis qui vivaient en Turquie ont dans leur quasi-totalité émigré en Allemagne dans les années 1980. On estime le nombre de Yézidis en Europe à 40000 (voir Kreyenbroek et Rashow, 2005, p. 5).

3. Originaires en majorité de la région de Gaziantep, les Yézidis de Transcaucasie ont fui les persécutions des musulmans et se sont dirigés vers le nord-est : d'abord Van et Kars, puis lors du recul des troupes tsaristes, dans le Caucase. 
4. Les Sheikhs et Pirs doivent participer aux rites de passage de leurs Mirid tels les naissances, mariages et funérailles. En échange les Mirid leur donnent de l'argent et leur montrent du respect.

5. Ceci constitue par ailleurs une politique voulue par l'État arménien.

6. Ne participent que les femmes mariées et mères, avec quelques rares exceptions pour les parents très proches.

7. Les Qewwl et Beyt sont deux types de prières chantées par les Sheikhs et Pirs lors de cérémonies.

8. Les femmes Sheikhs et Pirs, lorsqu'elles sont présentes, sont assises avec les femmes. Lorsque le défunt est de sexe féminin, elles ont pour rôle de laver le corps et préparer le linceul, mais en aucun cas elles ne récitent de Qewl et Beyt lors d'une cérémonie funèbre.

9. Extrait d'une lamentation enregistrée en studio et chantée par Sos Koçaryan.

10. Samo est le fils du défunt. La relation de parenté est soulignée.

11 Extrait d'une lamentation enregistrée en décembre 2005, à Sipan, lors des funérailles d'une femme âgée.

12. Extrait d'une lamentation chantée par Sheikh Xalo en septembre 2006, lors des funérailles de Razmik, mort d'un infarctus à Moscou et enterré au cimetière d'Alagyaz.

13. Référence au Kotel, rituel exécuté lors des funérailles d'un homme respecté. Les hommes sellent le cheval du défunt et le chargent des beaux habits, couteaux et fusils du défunt. Le cheval devancera le cercueil sur la route pour le cimetière. Le rituel de Kotel a quasiment disparu aujourd'hui dans les villages yézidis d'Arménie. Pour une description détaillée du rituel, voir Rudenko, 1982, p. 65-68.

14. Lamentation enregistrée à l'enterrement de Kerem, au village de Chamiran, en avril 2006.

15. Extrait d'une lamentation chantée dans les funérailles de Razmik, en septembre 2006. On peut relever que la lamentatrice qui est une nièce du défunt prend par ses paroles la place de la sœur du défunt (qui était assise à ses côtés).

16. Le mot $x e r i ̂ b$ a une palette de sens très étendue; il peut signifier l'exil, le fait d'être hors de chez soi, hors de son foyer, l'étrange, l'étranger, l'ennemi ou même la nostalgie profonde. Ce terme, d'origine arabe, est utilisé dans beaucoup de langues du Proche et du Moyen-Orient et il est fortement présent dans les poésies et chansons.

17. Extrait d'une lamentation chantée lors des funérailles de Razmik, en septembre 2006.

18. Extrait d'une lamentation enregistrée pour la mort de Valode Misto et Sebrie Kele décédés au Haut-Karabagh.

19. Arméniens originaires de Sassoun (Anatolie orientale).

20.Extrait d'une lamentation chantée par un Sheikh lors de funérailles à Sipan, en décembre 2005.
21. Le schéma mélodico-rythmique des lamentations, qui n'est pas spécifique aux funérailles, est présent dans toutes les régions kurdes (à la fois en Iran, Irak, Syrie et Turquie). Le modèle mélodique général des lamentations est composé d'une formule introductive ascendante, d'un récitatif sur une ou deux notes, et d'une formule conclusive descendante qui termine sur la note la plus grave de l'ambitus.

22. J'ai pu le constater à plusieurs reprises: je donnerai ici l'exemple de Chuchik, une femme de 60 ans vivant au village de Rya Teze dans la région d'Aparan. La veille de son départ pour Moscou où elle partait habiter chez son fils (son mari étant mort l'hiver précédent), son frère lui a demandé ce qu'elle voulait dire à ses amis en partant. Elle a immédiatement improvisé une lamentation d'exil qui a duré plus de 15 minutes, dans laquelle elle a expliqué toute sa situation. Elle a décrit la maladie et la mort de son mari, a exprimé ses inquiétudes sur la terre étrangère, Moscou, et l'exil. Ceci est certes une anecdote, mais cela nous indique que, dans certaines occasions il est plus adapté de s'exprimer en chant, en lamentation, en particulier pour les femmes (voir aussi Allison et Kreyenbroeck, 1996, p. 43).

23. Durant les trois jours de jeûne précédant le soir de Xidirnebi, des repas sont offerts par chaque famille aux voisins en mémoire des morts.

24. Durant la fête de Kiloç, un gâteau est partagé et distribué aux morts et aux vivants. 